\title{
INTEGRATING INFERENTIALISM ABOUT PHYSICAL THEORIES AND REPRESENTATIONS: A CASE FOR PHASE SPACE DIAGRAMS
}

\author{
JAVIER ANTA \\ Universitat de Barcelona \\ LOGOS \\ BIAP \\ antajav@gmail.com
}

SUMMARY: This paper argues for an integrated inferential conception about theories and representations and its role in accounting for the theoretical value of philosophically disregarded representational practices, such as the systematic use of phase space diagrams within the theoretical context of statistical mechanics. This proposal would rely on both inferentialism about scientific representations (Suárez 2004) and inferentialism about particular physical theories (Wallace 2017). I defend that both perspectives somehow converge into an integrated inferentialism by means of the thesis of theories as being composed of representations, as defended from the representational semantic conception defended by Suárez and Pero (2019).

KEY WORDS: inferential conception, scientific representation, phase portrait, statistical mechanics, semantic view

RESUMEN: En este trabajo defiendo una concepción inferencial integrada sobre teorías y representaciones y su papel en la explicación del valor teórico de prácticas de representación filosóficamente despreciadas, como el uso sistemático de diagramas de espacio de fase en el contexto teórico de la mecánica estadística. Esta propuesta se apoyaría tanto en el inferencialismo sobre las representaciones científicas (Suárez 2004) como en el inferencialismo sobre las teorías físicas particulares (Wallace 2017). Defiendo que ambas perspectivas convergen de alguna manera en un inferencialismo integrado mediante la tesis de las teorías como compuestas de representaciones, tal y como se defiende desde la concepción semántica representacional que Suárez y Pero (2019) defienden.

PALABRAS CLAVE: concepción inferencial, representación científica, retrato de fase, mecánica estadística, visión semántica

\section{Introduction}

It would be easy to see how a generalized, and sometimes even picturesque, idea of activity in the physical sciences in the collective imagination corresponds to that of an individual lucidly unraveling the foundations of a theory by simply manipulating symbols on a blackboard. However, the complex idiosyncrasies of real scientific 
practices imply an overwhelming dose of routine, the convergence of the technical and conceptual forces of a large number of hierarchically organized scientific agents, and the use of an enormous plurality of different and diverse resources (measurement apparatuses, images, conceptual schemes, diagrams, lines of code, and so on) employed to obtain meaningful knowledge about the phenomenon being studied. Concerning the latter, the philosophy of science throughout the twentieth century (with notable exceptions in its last decades, as seen in Hacking (1983) or Giere (1988)) has systematically and continuously disregarded the role of these "marginal" resources in both obtaining relevant knowledge and in the immediate configuration of theories. ${ }^{2}$ However, this lack of attention to what we might call "marginal representational practices' permeates philosophical reflection today.

In this paper I argue that a perspective focused on inferences about both representations and scientific theories can provide a satisfactory account of the role of this kind of marginal scientific practice. On the one hand, this position derives locally from an inferential conception of scientific representations such as the one developed by Suárez (2004), where representations must be properly understood from the possibility of exploiting them inferentially to extract relevant information about the represented phenomenon. On the other hand, in this conceptual perspective, the inferential conception of particular physical theories (like quantum mechanics or statistical mechanics) outlined by Wallace (2017), will also converge; this broadly claims that we should understand these theories as tools for drawing inferences about a particular physical domain. Furthermore, we will argue that both inferential conceptions could be integrated by assuming that scientific theories can be composed of representations, as recently defended by Suárez and Pero (2019) in their semantic representational conception of scientific theories. To illustrate the prospects of this integrated inferential view we will focus throughout this paper on evaluating the phase space diagrammatic formalism employed in statistical mechanics as a case study of representational

\footnotetext{
${ }^{1}$ We use the term 'marginal' to refer to those representational practices that have traditionally been conceived within the philosophy of science (although this has changed in recent decades, as we will point out later) as merely incidental or peripheral to the use of the theory from which they are performed.

${ }^{2}$ Although this changed at the end of the century, the cause of this disregard (following Giere's (2004) arguments) can be traced to the philosophical conception of scientific theories exclusively as sets of logical formulas or linguistic-like structures (the so-called syntactic conception), and as highly abstract mathematical-structural models, later. We will return to this topic in more detail in section 5 .
} 
practice. Finally, I argue for the value of this particular modelling apparatus (generally disregarded in the literature) in the understanding of the conceptual foundations of this physical discipline.

The plan for this paper is as follows. It begins by outlining the bases of statistical mechanics from the formalism of phase space, emphasizing the diagramming apparatus employed by Hemmo and Shenker (2012). Section 3 will be devoted to the philosophy of scientific representations, defending the inferential conception for its capacity to incorporate the sensitivity of these to the theoretical context and the role of epistemic agents. Additionally, the distinction between dynamical conceptions and inferential conceptions of physical theories outlined by Wallace (2017) will be explored, advocating for a non-epistemic version of the latter. Section 5 will present the semantic representational conception of Suárez and Pero (2019) as the position that allows us to robustly integrate inferentialism over representations and inferentialism over theories. Finally, this inferentialism of theories-representations will be applied to the particular case of statistical mechanical practices based on phase diagrams, showing the robust theoretical value of these representational practices.

\section{Statistical Mechanics and Phase Space Diagrams}

Before accounting for the role of phase diagrams within the theoretical context of statistical mechanics (henceforth 'SM'), at least some basic concepts must previously be clarified. First, SM is the physical discipline that studies the macroscopic behavior of certain systems with astronomical quantities of degrees of freedom (paradigmatically an ideal gas in a closed container) from the dynamic values, mainly position and momentum, of its components. The latter is called the 'micro-state' the exact determination of the position and moment of all components of the system at a specific time. The set of all possible values of position and momentum of the components of this system generates what is known as 'phase space'. Finally, a diagram or phase portrait would be a simplified graphical representation (as it compresses the information of a space of huge dimensions in a two-dimensional image) of the phase space of a system.

This paper argues that certain well-formed phase diagrams constitute robust representational devices that transmit statistical mechanical content in a valid and consistent way; this allows us to inferentially exploit them and obtain significant information about the target system. Therefore, we will now proceed to detail the basic elements of SM not only from the phase space but mainly with reference to its 
possible diagramming, in a way similar to how Hemmo and Shenker (2012) systematically employ phase portraits to expose the theoretical foundations of this discipline.

Technically, the phase space $\Gamma$ of a physical system $\mathrm{S}$ could be defined as an abstract $6 n$-dimensional vector space, where $n$ is the number of (microscopic, if $\mathrm{S}$ is a molecular system) components of $\mathrm{S}$, encoding position and velocity data about those components. Within this formal space, each individual phase point $\Gamma_{x}$ would represent an individual microstate of the physical system $\mathrm{S}$ where the position and velocity of all components are accurately determined. ${ }^{3}$ Due to the fact that the actual microstate of any realistic system with $n$ components (approximately $n=10^{24}$ ) would be epistemically inaccessible for pragmatic reasons, any phase space must irremediably incorporate the notion of probabilities as mathematical shortcuts that enable us to calculate and compute effectively how the macroscopically observable properties of the system will behave.

It should be noted that the introduction of statistical resources in the classical phase space is different in each of the two main approaches to SM, namely Boltzmann's formalism and Gibbs' formalism. ${ }^{4}$ In the Boltzmannian case, this is properly done by partitioning $\Gamma$ into different non-overlapping phase regions ${ }^{5} \Gamma_{M}$ corresponding to the different 'macrostates' of system $\mathrm{S}$, which are continuous sets of microstates to which are associated the value of some measurable 'macrovariable' (e.g. volume, pressure, temperature, and so on) of system S. The number (or more properly 'volume', as space $\Gamma$ is continuous in classical SM) of microstates contained in a macrostate is obtained by means of a particular measure, usually the Lebesgue measure. ${ }^{6}$ As for Gibbsian SM, wherein phase space $\Gamma$ is usually coarse-grained or partitioned into cells $\omega$ of equal phase volume, a

${ }^{3}$ As Shenker (2019) points out, the label 'micro' here refers neither to the size of the components (since S can also be a stellar or galactic system, as Robertson (2019) suggests) nor to the intuitive part-whole relationship between the components and the system, but to the fact that all position-momenta values of the constituents are 'completely determined' in this state.

${ }^{4}$ See Frigg 2008.

${ }^{5}$ We will use 'phase regions' as referring to both macrostates and phase blobs (dynamical evolutions of macrostates). Following Hemmo and Shenker's (2012) conventions.

${ }^{6}$ The most common justification for the use of the Lebesgue measure is by virtue of its conservation under the dynamics defined by the Liouville theorem. As Shenker (2019) points out, this is a mathematical or a priori justification, whose physical validity depends on its strict correlation with the relative frequencies of phase trajectories. 
probabilistic distribution $\Gamma_{\rho}$ over phase space is employed to represents not the physical system $\mathrm{S}$ itself but a collection or 'ensemble' of continuously infinite virtual copies of the system $\mathrm{S}$ having different microstatistical values.

Let us suppose now that we want to represent in phase-spatial terms a typical thermal behavior, paradigmatically the approximation of a molecular kinetic system to its thermal equilibrium state, as for example an ideal gas initially distributed in the left half of a vessel that begins to occupy its entire volume. That dynamic evolution of the gas $\mathrm{S}$ could be phase-spatially modelled in different ways depending on the SM formulation. For Boltzmannian SM, the state of system $\mathrm{S}$ at each instant of time $t$ is completely represented by a phase point $\Gamma_{x}(t)$, in this sense its Hamiltonian dynamic evolution during a certain time interval $\Delta t$ will generate a segment or phase path $\Gamma_{\gamma}(\Delta t)$ on the phase space, as depicted on the left diagram in Figure 1 below. On the other hand, from the perspective of Gibbsian SM fine-grained formalism, the dynamics of probability distribution $\Gamma_{\rho}$ is determined by Liouville's equation in the sense that it goes through the accessible phase space without changing its volume (according to Liouville's theorem) although it may change its shape in its dynamic evolution, as shown in the diagram on the right in Figure 1.

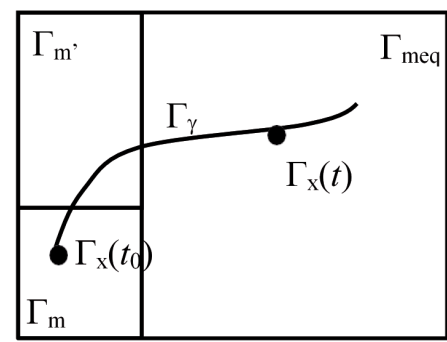

$$
\mu\left[\Gamma_{m}\left(t_{0}\right)\right]<\mu\left[\Gamma_{m e q}(t)\right]
$$

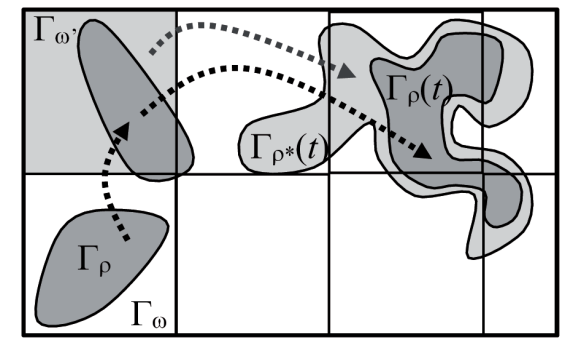

$\mu\left[\Gamma_{\rho}\left(t_{0}\right)\right]=\mu\left[\Gamma_{\rho}(t)\right] \mu\left[\Gamma_{\rho}\left(t_{0}\right)\right]<\mu\left[\Gamma_{\rho *}(t)\right]$

Figure 1. Phase portraits (also 'phase space diagrams') of a physical system approaching thermal equilibrium according to Boltzmann (left) and Gibbs (right) approaches to statistical mechanics.

From these basic mechanical-statistical resources we can now proceed to describe phase-diagrammatically how an ideal gas freely expands in a vessel according to each of the two main formalisms in SM. As 
Figure 1 (left) shows, for Boltzmann's proposal this behavior would be represented in phase space by a trajectory that 'typically' transits ${ }^{7}$ from a minuscule phase region $\Gamma_{m}$ (i.e. a low-probability macrostate) to the phase region with greatest volume $\Gamma_{m e q}$ (i.e. the most likely macrostate), assuming that the latter macrostate properly represents the state of thermal equilibrium ${ }^{8}$ of our target gas-vessel system. Additionally, since Boltzmann entropy $\mathrm{S}_{B}=\mathrm{k}_{B} \ln \mathrm{W}$ (wherein $\mathrm{k}_{B}$ is Boltzmann's constant) of a system would be proportional to the phase volume $\mathrm{W}$ associated with the macrostate containing the actual microstate of the system, a trajectory $\Gamma_{\gamma}$ (like the one depicted in Figure 1, left) approaching the phase region $\Gamma_{m e q}$ with largest phase volume would therefore represent an increase in Boltzmann's entropy during the free expansion of an ideal gas in a vessel.

On the other hand, for Gibbs' approach there are two different pictures (Figure 1, right) of how a gas approaches its equilibrium state in a free expansion. Firstly, for Gibbs' statistical mechanical framework it is not the physical system that reaches the state of thermal equilibrium, but the ensemble represented by the probability distribution $\Gamma_{\rho}$ that can reach its state of statistical or ensemble equilibrium. In this sense, an ensemble will be in the state of statistical equilibrium if $\Gamma_{\rho}$ is time-invariant under the dynamics of the system (Frigg 2008, p. 74). However, according to this definition, an ensemble could reach equilibrium even if its fine-grained entropy (proportional to the phase volume $\Gamma_{\rho}$, as well as to the area of $\Gamma_{\rho}$ in Figure 1, right) remains constant during the evolution according to the Liouville theorem seen previously, against the empirical predictions of the second law of thermodynamics. To solve this problem, Gibbs himself introduces a coarse-graining procedure (Uffink 2007), by which a new probability distribution $\Gamma_{\rho} *$ could be derived by phase-averaging the values of the initial distribution $\Gamma_{\rho}$ over the phase space cells $\omega_{i}$ (see Figure 1, right). Because the Gibbs entropy of $\Gamma_{\rho} *$ (whose distribution is uniform) will always be larger than that of $\Gamma_{\rho}$ at the same time $t$, the procedure of coarse-graining ensures that the entropy of the assembly increases according to the empirical principles of thermodynamics. This theoretical gap between Boltz-

${ }^{7}$ The typicality conception of the approach to thermal equilibrium is based on the assertion that the phase trajectory $\Gamma_{\gamma}$ will reach the macrostate of highest phase volume with an overwhelming degree of probability. However, due to the famous objections of reversibility and recursion (Uffink 2007; Frigg 2008), it is mechanically possible that a phase trajectory eventually returns to a macrostate with low phase volume.

${ }^{8}$ See Frigg 2008. 
mannian and Gibbsian conception of equilibrium are thoughtfully assessed in Werndl and Frigg (2017).

I conclude this section by pointing out what I will argue in the following sections, namely, that phase diagrams (Figure 1) are an excellent vehicle for transmitting the representational potential of SM, according to both Boltzmann and Gibbs' frameworks, as I have tried to show in the statistical mechanical modeling of the expansion of a gas in a vessel. However, we need certain conceptual tools to understand by virtue of which factors the components of the phase diagrams can come to represent phenomena such as the free expansion of an ideal gas. This is precisely the task on which we will focus next.

\section{An Inferential Conception of Phase Space Representations}

In the previous section I have discussed how phase space diagrams can be exploited to represent different features of a physical system with thermal behavior. This would lead us directly to two key questions, a general one about what scientific representations are, and a more specific one about how phase-space representations are to be understood within the theoretical context of SM. A superabundance of words have been written about the first question in the literature on philosophy of science in the last three decades, while the second one has not been treated in such a systematic way within the philosophical literature, despite its key role in understanding representational practices in SM. My aim in this paper is precisely to offer a satisfactory answer to the latter question. However, we cannot proceed otherwise than by evaluating the proposals derived from the general problem of scientific representation.

Many different philosophical treatments have recently emerged about how models, diagrams or equations (as well as other various resources) employed in scientific practices can come to represent the phenomena that these scientific areas deal with. All of this manifold of perspectives can be broadly classified according to how the relationship between that which is represented, or the representational source (models, diagrams, etc.), and the represented phenomenon or representational target (for example, an expanding gas) is characterized, both as constituents of the representation, ${ }^{9}$ see Figure 2.

${ }^{9}$ Note that the case illustrated in Figure 2 is particularly complex, as it represents a kind of double indirect representation. On the one hand, the distributions and * do not represent the gas as a physical system directly, but an assembly or collection of dummy copies of this system that encodes statistical properties on this system. 

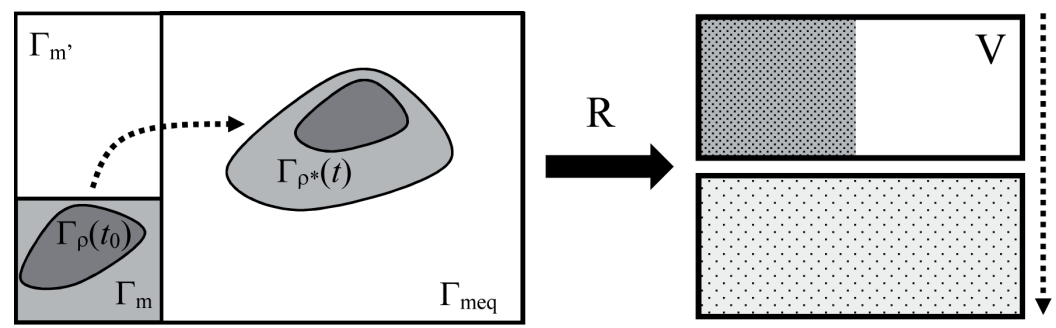

Figure 2. Is there an ' $R$ ' relationship between phase space structures (left) and a gas approaching thermal equilibrium at $\mathrm{V}$ (right) that robustly accounts for how the former elements can come to represent the latter physical phenomenon?

In this line we find two great paradigms about scientific representations: the substantivalist, where a deep or intrinsic ' $R$ ' relationship between source and target is postulated that would explain the representation; and the deflationary, where scientific representations cannot be explained on the basis of deep connections between the representational source and the representational target. One of the most relevant family of substantivalist conceptions within the philosophical literature is the one that claims that every relationship between the representing-source and the represented-target (' $R$ ' in Figure 2) is a structural relationship between both terms, a sort of formal map between the source and the representational target. This structural relation can be of several types, mainly: 'isomorphism', postulating a one-to-one correspondence between the elements of the source and the elements of the target; 'homeomorphism', mapping between the relational structure in the elements of the representational source and the relational structure in the elements of the represented; and 'partial isomorphism' (da Costa and French 2003), one-to-one correspondence between part of the elements of the representational source and the target. It is worth mentioning that we can also find non-structural substantivalist positions although, as Kuorikoski and Ylikoski (2015) defend, some of their candidate relations (eminently that of 'similarity' defended by Giere (2004)) are relatively vague and in no way contribute any explanatory value.

On the other hand, the phase portrait on the left is a two-dimensional diagrammatic representation (or simplification, if you will) of the complex multidimensional phase space. Without presupposing anything about transitivity between nested representations, we will assume from now on that phase spatial structures represent (with more or less success) the expanding gas, regardless of the degree of required mediation. 
As we will see below, one can argue that the success of these structuralist positions regarding scientific representations is directly linked to the predominance of the structural semantic conception of scientific theories (a theory is a set of formal and mathematical models) in the philosophical arena. But defending the representational role of mathematical structures through a structuralist perspective (e.g. Pincock (2012) or Bueno and Colyvan (2011)) is problematic. Concerning the case study assessed in this paper, the structuralist conception of phase space representations would assume that the explanatory contribution of the latter to the possibility of representing a physical phenomenon depends exclusively on the mathematicalformal character of these abstract structures (e.g. the symplectic structure of phase space). This would rule out the possibility of giving any representational contribution to the concrete non-mathematical resources linked to the diagrammatic expressions of phase space formalism, an idea that I will reject below.

Suárez (2004) carried out a critique of these structuralist positions appealing to the fact that the formal properties of the postulated intrinsic relations (isomorphism, homomorphism, etc.) contradicted the central properties that, intuitively, any relation that tries to explain a representation must have. In particular, the author states that while these structural relations are clearly symmetrical, the relationship between the representational source and the target represented is fundamentally asymmetrical. For example, some may find a sort of isomorphism or homeomorphism between certain phase space structures and an expanding gas in a container (Figure 2) (arguably a highly difficult and completely ad hoc task), but this structural relationship still does not explain the fact that phase space structures seek to represent the expanding gas and not the other way around.

However, we argue that the fact that these substantive-structural relationships do not meet certain formal requirements (asymmetry and others) that conform to our intuitions of what constitutes a representation does not explain their explanatory poverty when compared to scientific representations, in general, and to phase space representations in statistical mechanics, in particular. What explains this explanatory deficiency of substantivalism is the adoption of something exemplifying what in philosophy of language and mind is called 'semantic internalism' (Carter et al. 2017), where the capacity of a source to represent an external phenomenon depends exclusively on the constitutive or intrinsic properties of this representational source. Representational substantivalism as semantic internalism presupposes a robust and necessary connection between representational 
source and target system, between the phase space structures and the expanding gas of the above example. But it is easy to see that this is by no means the case. Let us suppose that those phase space structures in Figure 2 do not represent more or less successfully the thermal evolution of a gaseous system (a mole of helium) but that of (i) a self-gravitating star system (a disk galaxy) or (ii) a ferromagnet (a bar of iron) (Sklar 1993), where all can share the value of certain key macroscopic parameters, such as the number of components. Contrary to substantive conceptions, the compatibility of the same statistical mechanical resources to represent a wide range of physical phenomena (as recently shown by Robertson (2019) in the case of self-gravitational systems) would support in our particular case the thesis that there cannot be a deep or substantial connection between the phase spatial expression of these resources as representational sources and concrete physical phenomena.

Based on this argument, we would defend here a deflationary conception of scientific representations applied to the particular context of phase space formalism. Without going into the peculiarities of the different deflationary positions (maybe we should mention Hughes' 'DDI' model (1997) for its pioneering role), we will subscribe to the inferential framework about representations developed by Suárez (2004). In this paper, Suárez proposed two necessary conditions for a given support to constitute a representative source of a given representational objective. On the one hand, (i) there must be a 'directionality' (or representational force, in the author's terms) properly from what represents to the target represented, and not the other way around; that is, as phase diagrams are representations of the thermal behavior of molecular systems, it cannot be possible for the latter to represent the former. On the other hand, (ii) a competent agent could cognitively exploit the representational source to draw inferences about the representational objective. In this sense, scientific representations are conceived not properly by the relation between representational source and representational target but by the capacity to cognitively exploit the representational source so that an expert agent can infer relevant information about the phenomenon represented in a context of scientific practice:

We can then say that the relation $\mathrm{R}(a, b)$ constitutes the representation - or that it is the constituents of the representation - if and only if, for any (source, target) pair in any context, $\mathrm{R}$ (source, target) is the relation of representation. But, the relation $\mathrm{R}(a, b)$ is the means of the representation of $b$ by a in a particular context of use if and only if $\mathrm{R}(a, b)$ is 
the one relational property of $a$ and $b$ that is actively employed by the agent who, in the particular context, uses the representation in order to draw or infer conclusions about $b$ from $a$. (Suárez and Pero 2019, p. 355)

As the quotation shows, the relationship ' $R$ ' between representational source and target system would be only explanatory insofar as this ' $\mathrm{R}$ ' allows a cognitive agent within a delimited theoretical context to obtain information about a phenomenon (expanding gas) by means of inferential manipulation of the representational source (phase space structures), which is known in the literature as "subrogated reasoning'. ${ }^{10}$ Regarding the case study of this paper, what makes phase space diagrams a statistical mechanical representation of an expanding gas (Figure 2) according to Suárez's inferential conception is both the fact that (i) the former diagrams are representationally directed to the latter (and not the other way around) and (ii) that when exploited inferentially by a technical-theoretically competent scientific agent, it extracts physically relevant information about the thermal behavior of a gas.

Illustratively, when a competent agent, for example a graduate student in physics, looks at the phase diagram drawn on the left in Figure 2, he has the conceptual ability to understand that the two-dimensional area contained in phase-defined distributions as $\Gamma_{\rho}$ and $\Gamma_{\rho} *$ (representing statistical copies of a system) graphically encodes the N-dimensional phase volume of those probability densities. Starting from this preliminary point, from the theoretical context of fine-grained Gibbsian SM, our competent agent will be able to inferentially exploit the transition of the probability distribution $\Gamma_{\rho}\left(t_{0}\right)$ to $\Gamma_{\rho}(t)$ in such a diagrammatic representation (wherein area is preserved) to predict that the Gibbs entropy of the ensemble will be constant along the dynamic evolution of the system to which it is representatively directed. According to the inferential proposal (Suárez 2004), it is precisely this possibility of exploiting the diagrammatic resources in Figure 2 to infer or predict certain observable values of the macroscopic properties of a system that properly makes the former a representation of the latter.

Of course, a different matter is how the above constitutes a thermo-physically valid phase-diagrammatic representation, since it contradicts the empirical predictions of thermodynamics where entropy must increase or at least remain constant in the state of equilibrium (notice that the gas will only presumably reach a state of

${ }^{10}$ See Contessa 2007; Kuorikoski and Ylikoski 2015. 
equilibrium at the end of the expansion process). The theoretical procedure usually used from the Gibbsian framework to solve this problem is the one known as 'coarse-graining', where (let's suppose that at the moment $t_{0}$ ) the phase average of values in $\Gamma_{\rho}$ within the partition cell $\Gamma_{M}$ is performed, generating a new distribution of probability $\Gamma_{\rho} *$. Coarse-graining is chromatically encoded in Figure 2 by distinguishing between dark gray colored (fine-grained distributions) and light gray colored (coarse-grained) phase structures.

There is an extensive debate on whether coarse-graining is a theoretically significant process or not (Albert 2000; Frigg 2008), while it is often argued that the new distribution depends on the illusory or anthropomorphic nature of the partitioning of the phase space. Recently Robertson (2020) defended that the process of coarse-graining should not be understood as an illusory or anthropomorphic artifice, but that it would justify a process of abstraction from which a new autonomous statistical representation of the (epistemically inaccessible) dynamics of the system was obtained. In this respect, we would argue that Robertson's proposal (2020) can benefit greatly from Suárez's (2004) inferential conception. The main reason is that the status of representation as well as its epistemic potential is determined not by its capacity to mimic the actual microscopic dynamics of the gaseous system (maximizing the fine-graining of the statistical representation of the dynamic evolution of the system), as could be argued from any substantivalist conception of representations, but properly by the capacity to inferentially exploit this new coarse-grained distribution, for example, to give a satisfactorily theoretical account of how the irreversible thermal behavior of the molecular system can be derived from the reversible dynamics of the constituent molecules (Robertson 2020, p. 549).

Another explanatory advantage of inferentialism about representations in this context is that it can shed light from the two necessary conditions mentioned above on why certain elements of the phase space do not play any representational role. For example, recently McCoy (2020) argued that the computationally intractable individual microstates (points $\Gamma_{x}$ in phase space) do not faithfully represent the microscopic configuration of classical molecular systems. One reason for this meaninglessness is provided by the inferential conception: namely, that as long as the individual microstates cannot be inferentially exploited by statistically competent mechanical agents to develop statistical mechanical predictions (defended by Wallace (2017)), they do not meet the necessary condition (ii) to constitute representational sources of target properties. 
We must emphasize that despite introducing the agent as another element of representation, Suárez (2004, p. 772) defends that inferentialism delimits the objective character of scientific representations in their capacity to extract significant information from the objective phenomenon. The practices of the scientific community in which the representation is inserted will determine the mechanisms of reference fixation, delimiting whether a phase diagram refers to a gaseous process or to an autogravitational system (2004, p. 773). However, as far as the objective of this paper is concerned, the inferential conception is presented as suitable (as opposed to alternative conceptions, as we have seen) to account for the epistemic function of marginal representational practices in the philosophical literature, such as the spatial phase diagrams in SM. This suitability is reflected in the following factors (a) neutrality regarding the nature (material, formal-structural, abstract, etc.) of the constituents of the representations (Kuorikoski and Ylikoski 2015), (b) neutrality regarding the format (symbolic, iconic, diagrammatic, etc.) of the representations, and (c) sensitivity to practices linked to a theoretical context. From this position, a Gibbsian or diagram-based Boltzmannian representation (Figure 1) could be as representationally legitimate as a set of symbolic formulas (conditioned to their necessary representational directness and inferential extractability), although a different matter is in what sense it constitutes an effective representation: for instance, the particular ability of phase diagrams to graphically encode abstract statistical data could be much more limited than its formulaic counterpart. In any case, the inferential conception supposes a well-suited framework for dealing with those representational practices that are more marginal but no less important in the advance of science.

according to the inferential conception, scientific representation is, unlike linguistic reference, not a matter of arbitrary stipulation by an agent, but requires the correct application of functional cognitive powers (valid reasoning) by means that are objectively appropriate for the task at hand (i.e., by models that are inferentially suited to their targets). (Suárez 2004, p. 778)

\section{An Inferential Stance to Statistical Mechanics}

I have just argued for an inferential proposal about how a device like a phase space diagram in the theoretical context of SM might represent a target phenomenon like a gas free expansion of gas. In 
this section, we will explore an inferential perspective, developed independently of Suárez's proposal (2004), on how we should interpret locally the content of certain physical theories. Recently, Wallace (2017) defended the existence of two opposing attitudes that can be adopted not on the essence or general purpose but specifically on the interpretation-driven content of physical theories such as quantum mechanics or classical SM.

On the one hand, the 'dynamical conception' of physical theories assumes that the ultimate scientific purpose of these theories is to account for the dynamic behavior of physical systems independently of the epistemic resources of the agents. Thus, according to inferential stance, SM becomes a physical discipline that consists primarily of modelling statistically with the highest possible accuracy the dynamic values of the components that make up certain physical systems. On the other hand, the 'inferential conception' of physical theories interprets those very theories as necessary inferential tools required to extract relevant information about certain physical phenomena and their evolution. From this stance, one (if not 'the') central theoretical purpose of SM would be to extract macrostatistical values from a system on the basis of the macroscopic knowledge that an observer possesses about that target system, although its microscopic dynamics are not exhaustively fixed.

As Wallace himself (2017) defended, the philosophical relevance of his distinction between a dynamic and an inferential conception (although it would be more appropriate to speak of 'stances' or 'attitudes') of physical theories is not at all orthogonal to the debates about (i) the dependence of the theoretical content on the observing agent (objectivism versus subjectivism) or (ii) the reality of objective phenomena (realism versus instrumentalism). The main justification for philosophical use of these two attitudes or local conceptions of SM lies in the fact that they properly capture the subtleties of how statistical mechanical agents manage their theoretical resources in contexts of real scientific practice, and not merely in assuming philosophical theses about the direct or indirect role of knowledge or the dependence or independence of the scientific agent in the understanding of that physical theory.

In the same way, the dynamical vs. inferentialist debate in the context of the classical SM cannot be properly understood from the two main approaches to such theory, namely Boltzmann's and Gibbs's (section 2). Although the first one may seem inextricably dynamicist and the second one presents at first sight more affinity with inferen- 
tialism, there are important nuances. For example, within Boltzmannian SM the partition (or coarse-graining) of phase space could be easily interpreted from an inferential stance as a technical resource that allows the inferential exploitation of incomplete dynamic information. As is well known, Boltzmann originally introduced phase space partitioning (see left diagram on the left of Figure 1) and thus of probabilities in the face of the practical impossibility of calculating ${ }^{11}$ the microdynamics of an ideal gas ${ }^{12}$ closely linked to the impossibility of finding an analytical solution to Boltzmann's famous equation. In this sense, the introduction of macrostates within Boltzmann framework (Figure 1), encoding sets of observationally indistinguishable microscopic configurations, could be satisfactorily understood from the inferential conception as statistical structures that make it possible pragmatically-computationally, not to determine the exact microscopic dynamics of the system (dynamicist desideratum), but to carry out feasible mechanical statistical predictions.

Likewise, as Wallace (2017) himself demonstrated, the Gibbsian frame could also be understood from a strongly dynamicist perspective, shifting the focus from Hamiltonian to Liouvillian dynamics. Interestingly, one could render Robertson's (2020) justification of Gibbsian coarse-graining (see section 3) as a properly 'dynamicist justification', since such a procedure aims to derive a Liouvilleanlike dynamic of a well-behaved probability distribution $\rho$ (separating its relevant components from the non-relevant) that meets certain formal conditions specified in what the author calls the 'ZwanzigZeh-Wallace framework'. This framework, explicitly Gibbsian in its conceptual and technical foundations, allows the irreversible macroscopic behavior of the system to be described from its underlying reversible microdynamics. Therefore, Wallace (2017) showed, contrary to what is generally assumed, how Gibbsian formalism could be employed not only under an inferential perspective but also under an inferentialist conception. Then, both debates on the theoretical foundations of SM (dynamical-vs.-inferential, and Boltzmann-vs.-Gibbs) cannot be completely orthogonal. In fact, Wallace (2017) argues that the first debate can be much more clarifying regarding disagreements about the foundations of this discipline than the second, despite the enormous attention it has received in the literature. However, each

${ }^{11}$ In words of Shenker: "Suppose, by idealization, that we solve the equation of motion of the system [...] such a calculation is doubly impossible: the system is too complex, and the number of microstates, and hence of trajectory segment, is a continuous infinity" (2019, p. 11).

${ }^{12}$ See Uffink 2007. 
conception has its own deficiencies when it comes to illuminating the fundamental problems of SM:

\begin{abstract}
Classical SM seems to be a hybrid, displaying some features that suggest an inferential conception and some a dynamical one. Probability, in the classical deterministic context, is extremely difficult to understand dynamically. The time-asymmetric dynamics of the Boltzmann equation and its many relatives is extremely difficult to understand inferentially [...] I would end here with the suggestion that the inferentialistvs.-dynamicist way of understanding the debates is more helpful, and less prone to mutual miscommunication, than the Gibbs-vs.-Boltzmann approach currently prevalent. (p. 190)
\end{abstract}

At this point in my argument I suggest that, among the deficiencies mentioned in the above quotation, neither (i) a dynamicist conception nor (ii) a subjectivist or epistemic inferential interpretative stance could properly account for the disregarded role of phase diagrams (among other types of marginal representational practices) within SM. On the one hand, we find firstly what we might call an 'inclusion argument', wherein dynamicism (particular) conflates into inferentialism (general). Although dynamicism reduces its 'idealized' (Shenker 2019) pretension to computationally determine the microscopic dynamics of physically relevant systems, the very possibility of fixing its statistical or ensemble (or even 'Liouvillian') dynamics would be strongly conditioned to the very possibility of inferring statistically such dynamics. Therefore, the dynamical conception of SM would ultimately constitute just a borderline particular case of inferential stance centered on inferring microdynamic properties. Even if we consider this argument to be insufficient, from a dynamical perspective phase diagrams constitute highly deficient representational apparatuses when determining the micro or macrostatistical dynamics of a realistic gaseous system, since for this it would require diagrammatic apparatuses with an overwhelming number of graphical dimensions, with which it would immediately lose its pragmatic utility and its epistemic virtues (visualization, comprehensibility, etc.). In any case, the dynamical stance contributes absolutely nothing to our task of understanding the role of marginal representations, such as actual phase diagrams.

On the other hand, there are historical forms of inferentialism in SM, notably that of Jaynes (1957), ${ }^{13}$ who conceives such a discipline

${ }^{13}$ One of the most paradigmatic cases of SM-inferentialism in the history of ther- 
not as a theory about physical phenomena but about prediction techniques "To the inferentialist, virtually none of the claims made in SM are claims about the world in itself, but just how I should reason about the world given imperfect information" (Wallace 2017, p. 190). For example, the approximation of a gas to its thermal equilibrium state (Figure 1 and 2 ) is treated interpretatively as a process that modifies the agent's inferential capabilities, while the equilibrium state (or 'equilibrium ensemble') is not properly a state of a physical system but a state of the agent, where its macro-predictive capabilities (according to Jaynes (1957) are maximized. This undoubtedly unravels an important problem for locally interpreting a physical theory that aims to extract meaningful information about an objective domain. In section 6 we will argue how the inferential conception of representations by Suárez (2004) would illuminate in what sense the plausible 'objectivity' of this interpretative stance should be properly understood.

$\mathrm{Up}$ to this point, we can defend that the main virtue of the inferential stance is that it tells us what SM consists in (instrument to develop inferences), but it does not delimit the nature or format of the representational vehicles that should be properly used to make statistical inferences about the actual microstate or to predict how the probability density $\Gamma_{\rho}$ will evolve. In this sense, a phase diagram would be a theoretical instrument as legitimate as a system of symbolic formulas or a verbal description insofar as it could be exploited inferentially to obtain relevant mechanical statistical information. Indeed, as we have seen in the previous section, it would be possible to use a diagram (e.g. the right on in Figure 1) to predict that the probability density $\Gamma_{\rho}$ describing the ensemble under observation will be able to change its shape without modifying its phase volume (encoded in its depicted area) at moments after $t$, plausibly fibrillating over the accessible phase space. Therefore, Wallace's (2017) inferential conception of particular physical theories gives us the opportunity

mal physics is found in Edwin Jaynes' proposal (1957) based on the so-called 'Principle of Maximum Entropy'. The Jaynesian formalism is technically based on the application of information-theoretical tools (identifying Shannon's entropy measure with Gibbs' coarse-grained entropy) with the straightforward aim of maximizing the predictive capabilities of the observer concerning the evolution of the system's macrovariables. Jaynes defended a strongly epistemic interpretation of those probabilities used in the formalism of SM as the interpretative core of his proposal, where probabilities reflect the degree of belief of the observer about the variables in which these probabilities are defined. Conceptually, Jaynes SM approach should not be interpreted as a theory about the dynamics of thermophysical systems directly, but as a theory that deals with statistical mechanical inferences of agents. 
to rethink the place of marginal representations within theories (not only physical, but generally scientific), as we shall proceed to explore below.

\section{Defending the Semantic Representational Conception of Scientific Theories}

Summarizing, this paper has evaluated two types of inferential perspectives within the philosophy of SM. On the one hand, it has defended that, while the substantial and structural connections between phase-space structures and thermophysical phenomena do not explain the representational act, the phase space representations used in SM should be understood pragmatically from an inferential conception (such as the one defended by Suárez (2004)) that incorporates their dependence on the theoretical context and their inferential exploitability by an agent. On the other hand, we also have approached what Wallace (2017) calls the 'inferential conception' about particular physical theories, from which SM would be interpreted (without additional epistemic connotations) as a set of statistical modeling techniques that allow us to obtain inferentially relevant information about certain thermophysical phenomena. In this section we argue how both perspectives can converge in a philosophically fruitful way through the so-called semantic representational conception of scientific theories, recently defended by Suárez and Pero (2019), and the so-called 'Hughes-Giere-Suárez thesis'.

As is well known, at the dawn of logical positivism and even during the boom enjoyed by that conception during the 1950s and 1960s, scientific theories were conceived as sets of symbolic formulas generated from certain logical and axiomatically articulated languages. The transition from intellectual orthodoxy to the so-called 'semantic conception' of scientific theories since the 1960s is usually pointed to in the historiography of the philosophy of science as a broadly iconoclastic transition, when it constitutes the de facto transition from formulaic-formatted logical structures to more expressive mathematical structures (theoretical ensemble, theoretical model or state space), where both acquire semantic content through the interpretation of these formal elements. ${ }^{14}$ Note that for the syntactic and the semantic-structural conception of theories, the only representational vehicles capable of carrying theoretical content are the formulas of

\footnotetext{
${ }^{14}$ Note that this structuralist dogma, where both theories and their constituent models acquire semantic content through the interpretation of the structures that make them up, permeates the literature to this day (Pincock 2012).
} 
symbolic logic and of set/model theory, respectively. This is what we can call the 'formulaic-symbolic dogma' of the philosophy of science until the end of the 20th century.

A substantial change in this historical evolution took place from the 1980s onwards, when the fundamental role of diverse non-formalmathematical elements (experimental instruments, images, phase diagrams, conceptual schemes, and so on) began to be assumed in obtaining empirical knowledge and in the configuration of scientific theories (Hacking 1983; Giere 1988). In this context the concept of 'scientific models' began to capitalize on this plurality of scientific resources. ${ }^{15}$ This caused not only a shift of attention within the philosophy of science from theories as formulaic-formatted abstract structures to specific concrete models used in real scientific practices (devices from which information about certain phenomena is inferred), but a new way of conceiving theories beyond the misleading syntactic-semantic dichotomy. This new strongly pragmatic conception (initially linked to the so-called Stanford School of philosophy of science), especially the one developed by Cartwright (1999), meant assuming a continuity between the regulatory body of the theory and the set of scientific practices linked to that theory, where the use of 'peripheral' resources (models, instruments, images, schemes, etc.) would be included. However, according to the author, it is the latter and not the theoretical principles that play an active epistemic role (namely: descriptive, explanatory, predictive or in understanding) in obtaining knowledge.

It is at this point that the concept of 'scientific representation' begins (from the 1990s onwards) to gain relevance in the philosophical landscape, intuitively encompassing a greater generality of phenomena than the notion of 'model' and focusing on the problem of semantic content, as shown in Hughes' (1997) famous paper. Here we argue that the inferential conception of scientific representations allows us to account for the role of this manifold of resources without the need to establish a relative hierarchy between epistemically inactive theoretical principles (à la Cartwright 1999) and epistemically active peripheral representational resources, as the pragmatically-prone conception defends. Another of the philosophical advantages of the inferential conception of representations, against both the structuralist conception and against the pragmatic vision of theories, is that it allows us, on the one hand, to locate the theoretical content of

${ }^{15}$ See Frigg and Hartmann 2020. 
the peripheral representational resources and, on the other hand, to evaluate the epistemic contribution of such theoretical content.

Through the philosophical exploitation of this advantage and through the criticism of structuralist dogma (theories are composed of structural models), Suárez and Pero (2019) recently developed a semantic conception of scientific theories based on representations. The central thesis of the semantic representational conception of these authors is that theories are not made up of any structural models but of representations, assuming as a premise the Hughes-Giere-Suárez thesis in its main argument:

(P1) Theories are composed of models (Semantic Conception of Theories)

(P2) All models are representations (Hughes-Giere-Suárez Thesis)

(SR) Theories are composed of representations (Semantic Representational Conception)

The semantic representational conception (SR) of theories is a substantial novelty with respect to all existing conceptions in philosophical literature. On the one hand, SR confronts (i) the structuralist dogma by which theories are identified with logical (syntactic conception) or mathematical structures (semantic-structural conception) and (ii) the formulaic-symbolic dogma by which the only legitimate vehicles of theoretical content are symbolic formulas. On the other hand, SR also faces the epistemic hierarchies implicit in certain pragmatic positions, ${ }^{16}$ where only concrete model-representations and not general laws can have epistemic value. For the semantic representational conception, any type of representational vehicle, regardless of its nature (structural, abstract or concrete) and its format (symbolic, iconic or schematic), may be susceptible to possess theoretical content. However (as can be assumed from the inferential conception of representations) not just any representational structure can be a valid theoretical vehicle, since this requires a syntactically correct and semantically consistent coding of such content. For example, a mere arrangement of oranges on a table could not contain mechanical statistical content precisely because of its inability to code microstatistical values in a syntactically and semantically satisfactory way to obtain information about a thermal process.

Otherwise, a diagrammatic representation like the ones in Figure 1 and 2 would encode (although highly limited in its expressive power)

${ }^{16}$ See Frigg and Hartmann 2020. 
statistical mechanical content in a syntactically correct and semantically consistent fashion, since for example, by coherently modifying the shape but not the area of $\Gamma_{\rho}$ (Figure 1) we are directly manipulating the meaning of that graphical element according to SM, namely, that the coarse-grained entropy of $\Gamma_{\rho}$ can increase in the dynamical evolution of the system without contradicting Liouville's theorem. A competent agent of SM will be able to qualitatively predict certain macroscopic values of a gas from the fact that in the left diagram in Figure 2 the area of the fine-grain distribution $\Gamma_{\rho}$ remains invariant during its evolution, which depends intrinsically on the theoretical content encoded in this particular diagrammatic representation. Particularly, the mechanical-statistical meaning of Liouville's theorem would be visually manifested in the particular case of preserving the two-dimensional dark-grey area of $\Gamma_{\rho}$, wherein the content of such a theorem would be validly preserved from a nearly $10^{24}$-dimensional phase $_{\text {space }}{ }^{17}$ idealistically embedded in the two-dimensional graphical representation that the reader can visualize in Figure 2. In fact, from the framework of the representational semantic conception, correct-consistent apparatus such as phase diagrams would no longer be considered as marginal representational practices (as it would make sense to assert from the formulaic-symbolic dogma), but would be included in the body of the theory itself as a constitutive part of it. In this way, statistical mechanics as a theory would not be constituted by a set of claims displayed within a technical textbook, but rather by a set of valid representational practices with which to obtain significant information about a field of physical reality.

I conclude by pointing out that, while the semantic representational conception deals with the nature and constituent elements of scientific theories in general without regard to how we should understand their aims, Wallace's (2017) inferential and dynamical conceptions deal with how we should understand the purposes of particular physical theories without regard to their nature and constituent elements. As I will argue in the following section, both philosophical positions are not only complementary but also allow us to integrate Suárez's (2004) inferential conception of representations into a single perspective that proves extremely fruitful when it comes to understanding the theoretical role of the most diverse representational practices.

\footnotetext{
${ }^{17}$ Assuming that the physical system under consideration has a realistic number $\left(\mathrm{n}=10^{24}\right)$ of molecular components.
} 


\section{Integrating Inferentialism about Theories and Representations}

Once the SR proposal about theories has been displayed in the previous section, we now have all the conceptual resources required to integrate Wallace's (2017) inferentialism about physical theories and inferentialism about scientific representations à la Suárez (2004), and to use that integrated inferential conception to this paper's case study about phase space diagrams in SM. The core idea that I want to defend up to this point is that, from a semantic representational conception of theories, such as the one recently developed by Suárez and Pero (2019), the inferential stance about physical theories outlined by Wallace (section 4) and Suárez's inferential conception of representations (section 3) would fruitfully converge into an integrated and enhanced inferential conception of theories-representations (or equivalently, of 'theories-as-representations'). The basis of this proposal would be the following:

(A) Inferential Conception of Representations: Scientific representations should be understood from the capacity to infer information about phenomena from the cognitive manipulation of a representational source by a competent agent.

(B) Inferential Conception of (Physical) Theories: Particular theories should be understood as technical-conceptual tools developed to infer information about a certain domain.

(SR) Semantic Representational Conception: Theories are composed of representations.

Note that although SR postulates that theories are composed of representations, the former should not be understood as mere inarticulate collections of the latter. Each physical theory, like SM in our case study, constitutes a systematic integration of representational practices by a scientific community in a particular historical context with a certain domain (more or less flexible) of applicability and objectives with respect to other theories. In this sense, an inferential stay of physical theories (Wallace 2017) is not at all redundant with an inferentialism on representations (Suárez 2004) from our integral proposal. ${ }^{18}$ While (B) states directly that theories (e.g. SM) should

${ }^{18}$ By assuming that inferentialism about representations (A) can be derived from the conjunction of inferentialism about theories (B) and the representational semantic conception (SR), and that (B) is in turn derived from the conjunction of (A) and (SR), we would immediately be committing a fallacy of division and composition, respectively, concluding that the component parts (representations) have the very same properties as the composite (theories). 
be understood as inferential tools, (SR) would refine this statement in the sense of specifying that such tools are properly representational resources (phase diagrams), and through (A) we would understand these representational resources from their capacity to be inferentially exploited. This is precisely the function that each of the elements of our integral inferential proposal would fulfill. Then, our central thesis on which our integral inferential conception on theories-representations is articulated would be:

(C) Integrated Inferential Conception of Theories-Representations: Theories must be understood by the capacity to inferentially exploit the diverse representational resources that conform them by a community of competent agents with the purpose of obtaining significant information about the target phenomena.

We must point out that our inferential proposal is properly integral in the sense that each part contributes somehow positively to another of its parts, enhancing each of these philosophical or interpretative perspectives. Illustratively, the inferential conception of Suárez (2004) has the capacity to account for (if not already solve) the problem of the lack of objectivity pointed out by Wallace (2017) with respect to the inferential conception of physical theories. For example, one of the main problems of inferentialism in SM is that the temporal asymmetry we find in representations like the one in Figure 2 (where the probability distribution $\Gamma_{\rho}$ transits macrostates with increasing phase volume in the dynamical evolution of the gas system) should be properly understood as an asymmetry in the predictive capacities of the observer and not in the physical reality itself (Wallace 2017, p. 188). However, from Suárez's inferentialism about representations it could be argued that the lack of arbitrariness of the elements (Suárez 2004, p. 772) of the diagram encoding a certain temporal asymmetry (namely, that the probability distribution $\Gamma_{\rho}$ travels through macrostate-encoded rectangles having increasing area and not the other way around) would somehow be connected to the capacity of such representation to obtain significant information about a physically relevant temporal asymmetry in the represented process.

One of the main objectives of our inferential proposal is not to postulate a crystal clear mereological relationship between inferentiallyconceived theories and inferentially-conceived representations. On the other hand, we properly intend to straightforwardly emphasize that by grasping how certain representational practices work (in our 
case, phase diagrams) —a task associated with Suárez's inferentialism (A) - we are immediately in a better position to understand the conceptual and technical foundations of such a theoretical physics discipline (in our case, statistical thermal physics) in which such representations are inserted, a task associated in turn with Wallace's inferentialism (B). Additionally, we can argue at this point that opening up the range of valid representational resources that are relevant to a particular theory would largely contribute to obtaining new conceptual resources to deal philosophically with certain issues underlying this theory. For example, diagramming the process of coarse-graining (Figure 1) can help to understand more easily (as opposed to using alternative formulas) in what sense such a theoretical resource can become physically significant with respect to the underlying dynamics. ${ }^{19}$ In this sense, in the following section we will show the potential of our inferential proposal by evaluating the robust statistical mechanical content that a phase diagram can encode and in what sense this implies some form of epistemic contribution in the acquisition of statistical mechanical knowledge.

\section{Framing Phase Space Representations in Statistical Mechanics via Integrated Inferentialism}

Let us begin the application of our integrated inferential proposal on the theories and representations in the field of phase diagrams in statistical mechanics by considering a phase diagram similar to those used by Hemmo and Shenker (2012, p. 140) to illustrate (and arguably 'explain') a macroscopic measurement process used in the context of SM, depicted below in Figure 3. Initially, the phase space of a target system (e.g. an ideal gas) is partitioned into three different macrostates $\left(\Gamma_{m}, \Gamma_{m^{\prime}}\right.$ and $\left.\Gamma_{m e q}\right)$ each one associated with one particular value of a macroscopic observational variable, where the macrostate having the largest measurement $\mu\left(\Gamma_{\text {meq }}\right)$, graphically depicted as a two-dimensional area of the macrostates, properly corresponds to the state of thermal equilibrium of the system. As it constitutes a 'dynamic' phase diagram, the phase structures it contains synchronically represent three different moments in the evolution of the target system. In the initial moment $\left(t_{0}\right)$ we consider that macrostate $\Gamma_{m}$, the one furthest away from thermal equilibrium, has a positive and uniform probability measure (light grey colored) that statistically describes the system at moment $t_{0}$; this means that the

${ }^{19}$ See Robertson 2020. 
current micro-state of the system is found with equal probability at any of the points it contains.

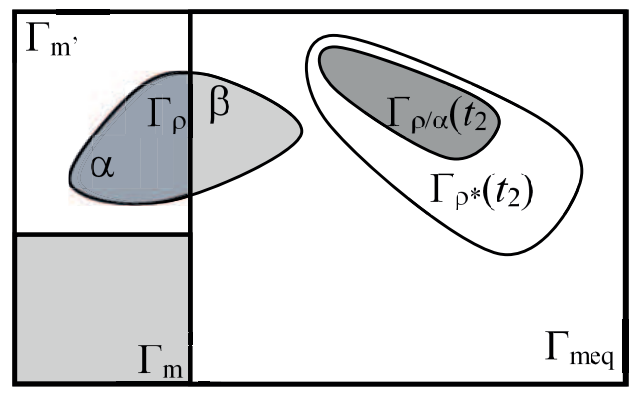

Figure 3. Phase space representation of the dynamic evolution of a physical system (mediated by a probabilistic assembly) in the theoretical context of classical statistical mechanics.

At the beginning of the dynamic evolution of the system, all the microstates contained in $\Gamma_{m}$ move along the phase space generating (let us say at time $t_{1}$ ) what Hemmo and Shenker call a 'dynamic blob' $\Gamma_{\rho}$ having a uniform distribution of probability defined over different regions in $\Gamma$. At this precise moment, a measurement would be carried out on the macrovariable associated with the macrostates, dividing the dynamic blob into two parts $\alpha$ and $\beta$ depending on the particular macrostate $\left(\Gamma_{m^{\prime}}\right.$ or $\Gamma_{m e q}$, respectively) in which they are found. Note that the partition of $\Gamma_{\rho}$ determines the probabilistic results of such measurement. Finally, let us imagine that the macrostatistical measurement results in the value of the macrovariable associated with $\Gamma_{m^{\prime}}$; then we take into consideration $\Gamma_{\rho / \alpha}$ (that is, the part contained in $\Gamma_{m^{\prime}}$, graphically highlighted in dark grey) and let it evolve dynamically until at the moment $t_{2}$ we would obtain $\Gamma_{\rho / \alpha}\left(t_{2}\right)$. On the other hand, we take $\Gamma_{\rho / \alpha}$ and carry out a phase averaging of its probability values along macrostate $\Gamma_{m^{\prime}}$ (namely, coarse-graining procedure as detailed in section 2) generating a new probability distribution $\Gamma_{\rho *}$ that will dynamically evolve into $\Gamma_{\rho *}\left(t_{2}\right)$.

Let us now apply our integrated inferential proposal as detailed in section 6. Firstly, we can point out how various graphic resources of the diagram serve to encode in a formal-syntactically correct and semantically consistent way statistical mechanical content. For example, the fact that the area contained in macrostate $\Gamma_{m}$ is uniformly light-grey colored can be considered as a graphic-chromatic resource 
used to encode that the probability distribution defined on that very macrostate will be uniform. Since any agent competent with (i) the syntactic-semantic functioning of this type of phase diagrams and (ii) with the basics concepts of SM will be perfectly able to access such graphically encoded theoretical content to draw inferences about the target system (e.g. concluding that any two possible microscopic configurations contained in this macrostate are equally likely to be the actual one), such a diagrammatic element (the uniform grey coloring of $\Gamma_{m}$ ) may be considered as a valid representational vehicle according to the inferential view (section 3 ).

In the same way, other graphic resources such as the area invariance between $\Gamma_{\rho / \alpha}$ at time $t_{1}$ and $\Gamma_{\rho / \alpha}$ at time $t_{2}$ would correctly and consistently encode the meaning of the Liouville principle as a mechanical statistical content (notice that if such an area were to change from $t_{1}$ to $t_{2}$, then the content of the Liouville theorem would be encoded in an incorrect and inconsistent way). Again, such a resource would be representationally significant as this element can be cognitively exploited by an agent to predict that the Gibbs entropy associated with the statistical ensemble represented by $\Gamma_{\rho / \alpha}$ will remain constant during the dynamical evolution of the system. From our inferential stance regarding $\mathrm{SM}$ as a systematic integration of a wide variety of representational devices, well-formed phase diagrams like the one in Figure 3 would properly offer, not an indispensable, but as an extremely effective tool for drawing statistical mechanical inferences (Wallace 2017) about the thermal behavior of certain kinetic systems.

Subsequently, our integrated-inferentialist analysis of the ability to inferentially exploit correctly and consistently encoded statistical mechanical content within phase diagrams also provides us with robust conceptual resources to assess the possible epistemic contributions of this particular type of representational practice. We argue that, although the inferential conception (Suárez 2004) originally does not determine the conditions by which a representation can be epistemically relevant or not, from our integral perspective we can delimit the way in which diverse representational resources (spatial phase in our case) contribute to the obtaining of knowledge (statistical mechanics in our case). In this sense it can be shown how phase diagrams not only have a merely pragmatic role (connected to their greater or lesser usefulness) in obtaining information about the objective thermal phenomenon, but constitutively epistemic.

Firstly, phase spatial diagrammatic representations increase the comprehensibility of the mechanical statistical content on which in- 
ferences are drawn because of its visualizability (or cognitive accessibility). For example, Liouville's theory is in principle more comprehensible by means of its visualizable diagrammatic representation (invariance of the area of $\Gamma_{\rho / \alpha}$ in Figure 3) than by means of the symbolic-analytical formula $\left|\Gamma_{\rho / \alpha}\left(t_{1}\right)\right|=\left|\Gamma_{\rho / \alpha}\left(t_{1}+\Delta t\right)\right|$, since any agent will require more technical and conceptual skills (as well as cognitive processing resources) to access the same statistical mechanical content from the first than from the second representation. Interestingly, this inferential exploitation of phase diagrams satisfies de Regt's (2017) definition of comprehensibility, by which an agent would understand the (e.g. mechanical statistical) content of the representation if it is able to derive qualitative information through cognitive manipulation of it.

Secondly, the fact that the phase diagram in Figure 3 assumes a valid (as well as correct and consistent) statistical mechanical representation (section 3) of a macroscopic measurement process of a free expanding gas indicates to us that it would be in principle possible to explain phase-diagrammatically certain statistical mechanical facts. For example, we can satisfactorily explain what a macroscopic measurement consists of through the valid representational resources contained in Figure 3, explicating that when performing a measurement at $t_{1}$ of an observable property (e.g. pressure) of the target system, the whole pre-measurement probability distribution $\Gamma_{\rho}$ collapses in either $\Gamma_{\rho / \alpha}$ or in $\Gamma_{\rho / \beta}$ with a degree of probability proportional to the graphically encoded area of each of these post-measurement distributions. Although the explanatory potential of phase space representations has been previously analyzed by Lyon and Colyvan (2008) within the nominalism-Platonism debate, we have gone one step further by showing how the capacity to exploit inferentially a phase diagram has a direct impact on the possibility of generating mechanical statistical explanations, for example, by making explicit how graphically separating the area of $\Gamma_{\rho}$ into two non-overlapping regions associated with macrostates $\Gamma_{m^{\prime}}$ and $\Gamma_{m e q}$ (respectively) at time $t_{1}$ could constitute a robust explanation of a macroscopic measurement process in SM.

Summarizing, our integrated inferential proposal has focused on the role of phase diagrams (usually conceived as ancillary or peripheral elements) as valid and robust statistical mechanical representations, due to their capacity to be inferentially exploited to obtain meaningful information through a correct and consistent encoding of statistical mechanical content. Furthermore, we have shown how 
phase diagrams constitute representational practices that not only contribute pragmatically but also epistemically to the inferential acquisition of statistical mechanical (being a constitutive element of this theory) knowledge by (i) encouraging theoretical comprehensibility or (ii) enabling diagrammatic explanations of target phenomena. Therefore, our integrated inferential conception developed here, constitutes a philosophical perspective that shows us, among others of its many virtues, how the theoretical potential (and therefore representational) of statistical mechanics goes beyond the mere manipulation of baroque analytical formulas or the entertainment of fuzzy mental models, thus showing us its highly disregarded but profound representational richness.

\section{Conclusion}

Let me go back over the parts of my main proposal as outlined during this paper. On the one hand, I explore how the inferential conception (Suárez 2004) satisfactorily accounts for scientific representations through the possibilities of being exploited inferentially to extract information about a target phenomenon. On the other hand, I have evaluated in what Wallace (2017) calls the 'inferential stance' about particular physical theories like SM, claiming that they could be properly understood by their usage of technical-conceptual tools to draw inferences. I defended how these two inferential perspectives could be integrated from a representational semantic conception, such as the one recently proposed by Suárez and Pero (2019), defending that scientific theories are properly constituted of representations. Once these elements have been set forth, I would defend what is the core of my argument: namely, that an integral inferential conception of theories-representations (or theories-as-representations) can shed light on the role of an enormous plurality of valid representational practices that constitute a theoretical context, wherein I would understand the latter more deeply through the analysis of the former.

In order to show its prospects, I have applied my integral inferential proposal to the representational use of phase diagrams in the context of statistical mechanics, evaluating how their graphic encoding of statistical mechanical content can be inferentially exploited to obtain meaningful information (in terms of explanation and understanding) about the target phenomenon. In this sense, my philosophical proposal has tried to show the robust role of phase diagrams in offering us a deep understanding of the issues surrounding statistical mechan- 
ics, unfairly disregarded in the literature despite their continued and systematic use by the main philosophers of statistical mechanics (e.g. Sklar 1993; Albert 2000; Frigg 2008; or Hemmo and Shenker 2012).

In any case, this has been only a concrete case study to show the virtues of my integral inferential conception. For obvious reasons of extension, I must leave for later works its capacity to shed light on the philosophical debates surrounding the topic I have dealt with here, such as (i) the key question (tangential to the debate on scientific realism) about whether the inferential exploitation of certain representational sources entails a certain ontological commitment by the agent with the entities denoted in those sources, or (ii) whether it is locally the representations, or globally the theory, that fixes the entities about which information is obtained through valid inferences. In any case, I will have to face these fascinating questions later on. ${ }^{20}$

\section{REFERENCES}

Albert, D., 2000, Time and Chance, Harvard University Press, Cambridge, Mass.

Bueno, O. and M. Colyvan, 2011, "An Inferential Conception of the Application of Mathematics", Noûs, vol. 45, no. 2, pp. 345-374.

Carter, A., J. Collin and O. Palermos, 2017, "Semantic Inferentialism As (A Form of) Active Externalism", Phenomenology and the Cognitive Sciences, vol. 16. no. 3, pp. 387-402.

Cartwright, N., 1999, The Dappled World: A Study of the Boundaries of Science, Cambridge University Press, Cambridge. (https://doi.org/10. 1017/CBO9781139167093)

Contessa, G., 2007, "Scientific Representation, Interpretation, and Surrogative Reasoning", Philosophy of Science, vol. 74, no. 1, pp. 48-68.

da Costa, N.C.A. and S. French, 2003, Science and Partial Truth: A Unitary Approach to Models and Scientific Reasoning, Oxford University Press, USA.

de Regt, H., 2017, Understanding Scientific Understanding, Oxford University Press, USA.

Frigg, R., 2008, "A Field Guide to Recent Work on the Foundations of Statistical Mechanics", in Dean Rickles (ed.), The Ashgate Companion to Contemporary Philosophy of Physics, Ashgate, London, pp. 991-996.

${ }^{20}$ I thank the anonymous reviewers for their really useful comments and suggestions. This work has been funded by an FPU Grant (FPU16/0774) of the Spanish Ministry of Education. It has been developed under the research framework of LOGOS, BIAP and the research group "Laws, explanation and realism in physical and biomedical sciences" (FFI2016-76799-P). 
Frigg, R. and S. Hartmann, 2020, "Models in Science", in Edward N. Zalta (ed.), The Stanford Encyclopedia of Philosophy, downloaded from <https://plato.stanford.edu/archives/spr2020/entries/models-science/> [accessed: 13/03/2021].

Giere, R., 2010, "An Agent-Based Conception of Models and Scientific Representation”, Synthese, vol. 172, no. 2, pp. 269-281. (https://doi.org/10. 1007/s11229-009-9506-z)

— 2004, "How Models Are Used to Represent Reality", Philosophy of Science, vol. 71, no. 5, pp. 742-752.

- 1988, Explaining Science: A Cognitive Approach, University of Chicago Press, Chicago, IL.

Hacking, I., 1983, Representing and Intervening: Introductory Topics in the Philosophy of Natural Science, Cambridge University Press, Cambridge.

Hemmo, M. and O. Shenker, 2012, The Road to Maxwell's Demon, Cambridge University Press, Cambridge.

Hughes, R.I.G., 1997, "Models and Representation", Philosophy of Science, vol. 64, no. 4, pp. S325-S336.

Jaynes, E.T., 1957, "Information Theory and Statistical Mechanics", Physical Review, vol. 106, no. 4, pp. 620-630.

Kuorikoski, J. and P. Ylikoski, 2015, "External Representations and Scientific Understanding", Synthese, vol. 192, no. 12, pp. 3817-3837.

Lyon, A. and M. Colyvan, 2008, "The Explanatory Power of Phase Spaces", Philosophia Mathematica, vol. 16, no. 2, pp. 227-243.

McCoy, C.D., 2020, “An Alternative Interpretation of Statistical Mechanics", Erkenntnis, vol. 85, no. 1, pp. 1-21.

Parker, D., 2011, "Information-Theoretic Statistical Mechanics without Landauer's Principle", British Journal for the Philosophy of Science, vol. 62 , no. 4, pp. 831-856.

Pincock, C., 2012, Mathematics and Scientific Representation, Oxford University Press, USA.

Robertson, K., 2020, "Asymmetry, Abstraction, and Autonomy: Justifying Coarse-Graining in Statistical Mechanics", British Journal for the Philosophy of Science, vol. 71, no. 2, pp. 547-579.

— 2019, "Stars and Steam Engines: To What Extent Do Thermodynamics and Statistical Mechanics Apply to Self-Gravitating Systems?", Synthese, vol. 196, no. 5, pp. 1783-1808.

Shenker, O., 2019, "Information vs. Entropy vs. Probability", European Journal for Philosophy of Science, vol. 10, no. 1, pp. 1-25.

Sklar, L., 1993, Physics and Chance: Philosophical Issues in the Foundations of Statistical Mechanics, Cambridge University Press, Cambridge.

Suárez, M., 2004, "An Inferential Conception of Scientific Representation", Philosophy of Science, vol. 71, no. 5, pp. 767-779. (https://doi.org/10. 1086/421415)

Suárez, M. and F. Pero, 2019, "The Representational Semantic Conception", Philosophy of Science, vol. 86, no. 2, pp. 344-365. 
Uffink, J., 2007, "Compendium of the Foundations of Classical Statistical Physics", in J. Butterfield and J. Earman (eds.), Handbook for Philosophy of Physics, Elsevier, Amsterdam, downloaded from <http://philsciarchive.pitt.edu>

Wallace, D., 2017, "Inferential vs. Dynamical Conceptions of Physics", in O. Lombardi, S. Fortin, F. Holik, C. López (eds.), What Is Quantum Information?, Cambridge University Press, Cambridge, pp. 179-204.

Werndl, C. and R. Frigg, 2017, "Mind the Gap: Boltzmannian versus Gibbsian Equilibrium", Philosophy of Science, vol. 84, no. 5, pp. 1289 1302.

Received: January 11, 2021; accepted: March 20, 2021. 